\title{
Association of toll-like receptor 9 expression with prognosis of systemic lupus erythematosus
}

\author{
YI YUAN $^{1,2}$, LING ZHAO ${ }^{1}$, ZHUANG YE ${ }^{1}$, HONGSHUANG MA ${ }^{1}$, XIAOSONG WANG ${ }^{2}$ and ZHENYU JIANG ${ }^{1}$ \\ ${ }^{1}$ Department of Rheumatology and Immunology; ${ }^{2}$ Institute of Translational Medicine, \\ The First Hospital of Jilin University, Changchun, Jilin 130021, P.R. China
}

Received November 25, 2016; Accepted July 20, 2017

DOI: $10.3892 /$ etm.2019.7290

\begin{abstract}
The current study assessed the association between toll-like receptor 9 (TLR9) and systemic lupus erythematosus (SLE) and subsequently determined the predictive value of TLR9 in assessing the prognosis of SLE. A total of 90 newly diagnosed patients with SLE and 49 healthy control subjects were enrolled in the current study. The expression of TLR9 mRNA was measured in whole blood samples from patients and controls. All patients were followed up for $\geq 2$ years and their clinical parameters were recorded. After 2 years, 30 patients were randomly chosen from patient subgroups with high $(n=20)$ or low $(n=10)$ TLR9 levels and the expression of TLR9 mRNA were measured again. Cox proportional hazards regression was used to identify the risk factors of SLE prognosis. Patients with SLE and high SLE disease activity exhibited significantly increased TLR9 expression $(\mathrm{P}<0.05)$. Persistent proteinuria of $>0.5 \mathrm{~g} /$ day [hazard ratio (HR), 6.314; 95\% confidence interval (CI), 2.858-13.947], C-reactive protein levels (HR, 1.013; 95\% CI, 1.007-1.019) and high-TLR9 mRNA expression (HR, 3.852; 95\% CI, 1.931-7.684) were independent risk factors of poor prognosis during a 2-year follow-up period, whereas patient treatment with $>1$ immunosuppressant (HR, 0.374; 95\% CI, 0.173-0.808) was a factor indicating favorable prognosis. Furthermore, the expression of TLR 9 mRNA remained high in patients with poor prognosis at the end of a 2-year follow-up period but in patients with a favorable prognosis, TLR9 mRNA expression was significantly reduced compared with the levels measured at SLE onset $(\mathrm{P}<0.0001)$. Therefore, the expression of TLR9
\end{abstract}

Correspondence to: Dr Zhenyu Jiang, Department of Rheumatology and Immunology, The First Hospital of Jilin University, 71 Xinmin Street, Changchun, Jilin 130021, P.R. China E-mail: jlujzy@163.com

Dr Xiaosong Wang, Institute of Translational Medicine, The First Hospital of Jilin University, 71 Xinmin Street, Changchun, Jilin 130021, P.R. China

E-mail: zhuanhuawang@163.com

Key words: systemic lupus erythematosus, toll-like receptor 9, prognosis
mRNA in whole blood samples at SLE onset is associated with SLE disease activity and its expression may be used as an indicator of poor prognosis in patients with SLE.

\section{Introduction}

Systemic lupus erythematosus (SLE) is a chronic autoimmune disease with a variety of clinical outcomes, ranging from benign illness to progressive disease, which may result in organ failure and mortality (1). The heterogeneity of SLE makes the prediction of patient prognosis challenging. The majority of previous studies investigating SLE prognosis have focused on assessing its clinical manifestation and parameters (2-4). At present, high levels of anti-double stranded DNA antibody titers (dsDNA $\mathrm{Ab})$, hypocomplementemia and renal dysfunction are the most reliable predictors of SLE prognosis (5-7). However, there are currently no biomarkers that accurately predict SLE prognosis.

Toll-like receptor 9 (TLR9) is a transmembrane receptor widely expressed in the monocytes and $\mathrm{T}$ and $\mathrm{B}$ cells of patients with SLE $(8,9)$. TLR9 is a receptor for extracellular DNA and is therefore capable of nucleic acid recognition (10). Although the role of TLR9 in SLE remains controversial, the involvement of TLR9 in the pathogenesis of SLE by identifying DNA-containing immune complexes has been established (11). It has been demonstrated that the expression of TLR9 mRNA is associated with SLE disease activity (12) and high SLE disease activity (including high SLEDAI score and severe organ damage) is associated with poor patient prognosis (13-15). It has been demonstrated that high disease activity at the time of SLE diagnosis does not prevent remission in patients with SLE (16). Thus, the association between TLR9 and SLE disease activity and the predictive value of TLR9 in SLE prognosis requires further investigation.

The current study aimed to determine TLR9 expression in patients with newly diagnosed and active SLE and investigate the associations between TLR9 expression and the clinical parameters of SLE. Furthermore, the potential of using TLR9 as a predictor of SLE prognosis was determined over a 2-year period.

\section{Materials and methods}

Patients and collection of data. A total of 90 patients with newly diagnosed SLE (87 females and 3 males, mean age at 
enrollment, $38 \pm 13.9$ years) were enrolled in the current study at the Department of Rheumatology and Immunology of the First Hospital of Jilin University (Changchun, China) between October 2013 and August 2014. All patients with SLE were followed up until August 2016. A total of 49 age- and sex-matched healthy controls (47 females, 2 males, mean age at enrollment, $36.8 \pm 12.8$ years) were enrolled from the Medical Examination Center of the First Hospital of Jilin University between October 2013 and January 2014 and acted as a control group.

The inclusion criteria for enrollment was newly diagnosed SLE according to the American College of Rheumatology (ACR) revised criteria for the classifications of SLE (17). The exclusion criteria were as follows: i) Use of steroids, antimalarial drugs or immunosuppressants prior to diagnosis and ii) the presence of active infection, hypertension, diabetes or neoplastic disease at the time of SLE diagnosis. Clinical and laboratory parameters were recorded during each visit, according to a standardized protocol (18) and the clinical manifestations of SLE were defined using definitions outlined by the ACR (17).

The current study was approved by the Institutional Medical Ethics Review Board of the First Hospital of Jilin University. Written informed consent was obtained from all patients in compliance with the Declaration of Helsinki.

Disease activity and definitions. Disease activity was monitored using the SLE Disease Activity Index (SLEDAI)-2K (19) and calculated at each visit. Patients were divided into SLEDAI $\leq 9$ and SLEDAI $>9$ groups, according to the SLEDAI-2K index. Due to the limited number of kidney biopsies available from patients, 'renal involvement' in the disease was defined as persistent proteinuria of $>0.5 \mathrm{~g}$ /day with no infection (19). The Crithidia luciliae indirect immunofluorescence test is the typical method used to measure levels of anti-dsDNA antibodies (Abs) in the clinic (20) and was used in the current study as it is regarded as a reference method due to its high specificity (21). An anti-dsDNA $\mathrm{Ab}$ of $\geq 1: 100$ was considered to indicate high-dsDNA Ab whereas $\leq 1: 32$ was defined as a low-dsDNA Ab. Patients were categorized into two groups during the 2-year follow-up period: A favorable prognosis group and a poor prognosis group, according to disease activity. Patients in the favorable prognosis group had an SLEDAI-2K <4; an active serological profile consisting of a low complement C3 levels (defined as C3 level <0.9 g/l; normal range is 0.9-1.8 g/l) and/or high anti-dsDNA Ab levels; were defined as clinically quiescent, serologically active or quiescent $(22,23)$ during each visit over the consecutive 2-year period and were treated with a daily dose of prednisone ( $<5 \mathrm{mg}$ ), immunosuppressants (cyclophosphamide, mycophenolate, cyclosporine, methotrexate, azathioprine or leflunomide) and antimalarials. Poor prognosis was defined as situations other than favorable prognosis, including mortality, persistent active disease (PAD; defined as SLEDAI-2K $\geq 4$ excluding serology alone, on $\geq 2$ consecutive visits) (24) and flare. Flare was defined as the presence of $\geq 1$ of the following features: i) $>3$-point change in the SLEDAI score, ii) new or worsening clinical lupus symptoms and iii) an increase in the steroid dose (25) during follow-up visits.

Treatment and follow-up. All 90 patients with SLE were treated with $0.5 \mathrm{mg} / \mathrm{kg} /$ day methylprednisolone (Pfizer, Inc.,
New York, NY, USA) and $400 \mathrm{mg} /$ day hydroxychloroquine (Sanofi-Synthelabo Ltd., Guildford, UK). A proportion of patients also underwent treatment with immunosuppressants, including cyclophosphamide (100 mg on alternate days; Maoxiang Pharmaceutical Co., Ltd., Tonghua, China), mycophenolate $(25 \mathrm{mg} / \mathrm{kg} /$ day; Roche Diagnostics $\mathrm{GmbH}$, Mannheim, Germany), cyclosporine (3 mg/kg/day; North China Pharmaceutical Group Corp., Hebei, China), methotrexate (10-15 mg/week; Xinyi Pharmaceutical Co., Ltd., Shanghai, China), azathioprine (50 mg, twice/day; Excella $\mathrm{GmbH}$, Feucht, Germany) or leflunomide (20 mg/day; Xinkai Pharmaceutical Co., Ltd., Suzhou, China) (Table I).

All patients with SLE were followed-up until August 2016 and evaluated for clinical manifestations and laboratory parameters during $\geq 4$ annual visits over the 2-year follow-up period. Situations in which patients were classified as having 'poor prognosis' were as follows: i) Patients who succumbed, ii) patients that experienced a flare, of which the date was assumed to be the midpoint between the visit at which an SLE flare was initially diagnosed and the previous follow-up visit and iii) patients with PAD, in which the date was defined as the midpoint between the first visit at which SLE patients exhibited PAD and the previous follow-up visit.

All 90 patients with SLE were divided into two groups according to the level of TLR9 mRNA expression at SLE onset. The high-TLR9 group included patients with a TLR9 mRNA level higher than the mean TLR9 mRNA level in healthy controls + two standard deviations. The remaining patients were classified as the low-TLR9 group. Additionally, 30 patients with SLE (20 from the low-TLR9 group and 10 from the high-TLR9 group) were randomly selected using a random number table to measure TLR9 mRNA expression 2 years following the induction of immunosuppressive treatment.

Blood sample collection and reverse transcription-quantitative polymerase chain reaction ( $R T-q P C R)$. Peripheral venous blood samples $(2 \mathrm{ml})$ were collected from all patients prior to and 2 years following the induction of immunosuppressive treatment. Following centrifugation $\left(4^{\circ} \mathrm{C}, 1,000 \mathrm{x} \mathrm{g}, 10 \mathrm{~min}\right)$, whole blood samples were suspended in $1 \mathrm{ml}$ TRIzol reagent (Invitrogen; Thermo Fisher Scientific, Inc., Waltham, MA, USA) and stored at $-80^{\circ} \mathrm{C}$. Total RNA was isolated from whole blood using TRIzol. The purity of RNA was determined using a Synergy ${ }^{\mathrm{TM}}$ H1 Hybrid Reader (BioTek China, Beijing, China). RNA samples were reverse transcribed into cDNA using a PrimeScript RT Reagent kit (Takara Biotechnology Co., Ltd., Dalian, China). The levels of TLR9 mRNA relative to GAPDH were determined by qPCR using a SYBR Green PCR Master Mix kit and specific primers on an ABI 7500 Real-Time PCR system (Applied Biosystems; Thermo Fisher Scientific, Inc.). The primer sequences used were as follows: TLR9 forward, 5'-CTGCCTTCCTACCCTGTGAG-3' and reverse, 5'-GGA TGCGGTTGGAGGACAA-3'; GAPDH forward, 5'-CGGATT TGGTCGTATTGGG-3' and reverse, 5'-TCTCGCTCCTGG AAGATGG-3'. Samples were incubated at $95^{\circ} \mathrm{C}$ for $10 \mathrm{~min}$, followed by 40 cycles of denaturation at $95^{\circ} \mathrm{C}$ for $10 \mathrm{sec}$ and annealing and extension at $60^{\circ} \mathrm{C}$ for $30 \mathrm{sec}$. The reactions were performed in duplicate. Relative TLR 9 mRNA expression was calculated using the $2^{-\Delta \Delta \mathrm{Cq}}$ method (26). 
Table I. The demographic and clinical characteristics and therapies administered to the high-TLR9 and low-TLR9 groups at baseline.

\begin{tabular}{|c|c|c|c|}
\hline Characteristics & Low-TLR9 group & High-TLR9 group & P-values \\
\hline TLR9 mRNA levels, median (LQ, UQ) & $18,402(10,687,23,734)$ & $40,450(32,510,49,442)$ & $<0.0001$ \\
\hline Patients, $\mathrm{n}$ & 67 & 23 & \\
\hline Age, years & $40.6 \pm 13.5$ & $30.4 \pm 12.4$ & \\
\hline Female, N (\%) & $66(98.5)$ & $23(100)$ & n.s. \\
\hline Lag-time onset-diagnosis, months & $16.8 \pm 16.3$ & $17.1 \pm 15.2$ & n.s. \\
\hline SLEDAI & $13.7 \pm 9.1$ & $17.4 \pm 10.6$ & n.s. \\
\hline \multicolumn{4}{|l|}{ Clinical manifestations } \\
\hline Fever, n $(\%)$ & $25(37.3)$ & $10(43.5)$ & n.s. \\
\hline Skin involvement, n (\%) & $32(47.8)$ & $11(47.8)$ & n.s. \\
\hline Arthritis, n (\%) & $43(64.2)$ & $16(69.6)$ & n.s. \\
\hline Serositis, n (\%) & $19(29.7)$ & $7(30.4)$ & n.s. \\
\hline Persistent proteinuria of $>0.5 \mathrm{~g} /$ day, $\mathrm{n}(\%)$ & $23(34.3)$ & $12(52.2)$ & n.s. \\
\hline Neuropsychiatric manifestations, n (\%) & $5(7.5)$ & $6(26.1)$ & 0.029 \\
\hline Vasculitis, n (\%) & $13(19.4)$ & $4(17.4)$ & n.s. \\
\hline Haematological involvement, n (\%) & $30(44.8)$ & $15(65.2)$ & n.s. \\
\hline \multicolumn{4}{|l|}{ Laboratory parameters } \\
\hline White blood cell counts & $5.8 \pm 3.6$ & $5.7 \pm 3.3$ & n.s. \\
\hline Red blood cell counts & $3.8 \pm 0.7$ & $3.3 \pm 0.7$ & n.s. \\
\hline Platelet counts & $163.3 \pm 90.5$ & $168.1 \pm 95.9$ & n.s. \\
\hline $\operatorname{IgM}$ & $1.3 \pm 0.7$ & $1.1 \pm 0.6$ & n.s. \\
\hline $\operatorname{IgA}$ & $3.2 \pm 1.5$ & $2.8 \pm 1.5$ & n.s. \\
\hline $\operatorname{IgG}$ & $17.6 \pm 9.1$ & $18.8 \pm 9.3$ & n.s. \\
\hline CRP, median (LQ, UQ) & $3(1,18.3)$ & $4(1,12)$ & n.s. \\
\hline ESR & $42.6 \pm 35.2$ & $35.4 \pm 35.1$ & n.s. \\
\hline Serum C3 level & $0.71 \pm 0.31$ & $0.55 \pm 0.28$ & n.s. \\
\hline Serum C4 level & $0.13 \pm 0.13$ & $0.09 \pm 0.06$ & n.s. \\
\hline Low C3 serum level, n (\%) & $49(73.1)$ & $20(87)$ & n.s. \\
\hline Low C4 serum level, n (\%) & $41(61.2)$ & $14(60.9)$ & n.s. \\
\hline ANA positivity, n (\%) & $67(100)$ & $23(100)$ & n.s. \\
\hline Anti-dsDNA Ab positivity, n (\%) & $49(73.1)$ & $20(87.0)$ & n.s. \\
\hline \multicolumn{4}{|l|}{ Drugs } \\
\hline Glucocorticoids, n (\%) & $67(100)$ & $23(100)$ & n.s. \\
\hline Hydroxychloroquine, n (\%) & $67(100)$ & $23(100)$ & n.s. \\
\hline \multicolumn{4}{|l|}{ Immunosuppressants } \\
\hline Cyclophosphamide, n (\%) & $13(19.4)$ & $5(21.7)$ & n.s. \\
\hline Mycophenolate, n (\%) & $29(43.3)$ & $14(60.9)$ & n.s. \\
\hline Cyclosporine, n (\%) & $0(0)$ & $8(34.8)$ & $<0.0001$ \\
\hline Methotrexate, n (\%) & $0(0)$ & $2(8.7)$ & n.s. \\
\hline Azathioprine, n (\%) & $1(0)$ & $1(2.8)$ & n.s. \\
\hline Leflunomide, n (\%) & $6(11.1)$ & $2(5.6)$ & n.s. \\
\hline >1 immunosuppressant, $\mathrm{n}(\%)$ & $11(16.42)$ & $4(17.39)$ & n.s. \\
\hline \multicolumn{4}{|l|}{ Prognosis } \\
\hline Mortality rate, n (\%) & $2(3.0)$ & $4(17.4)$ & 0.035 \\
\hline Poor prognosis over 2 -years, $\mathrm{n}(\%)$ & $22(32.8)$ & $16(69.6)$ & 0.003 \\
\hline
\end{tabular}

All results are presented as the mean \pm standard deviation, unless otherwise specified. $\mathrm{P}<0.05$ was considered to indicate a statistically significant difference. SLEDAI, systemic lupus erythematosus disease activity index; n, number; n.s., no significance; LQ, lower quartile; UQ, upper quartile; TLR9, toll-like receptor 9; Ig, immunoglobulin; CRP, C reactive protein; ESR, erythrocyte sedimentation rate; dsDNA, double stranded DNA; ANA, antinuclear antibody; AB, antibody titer. 

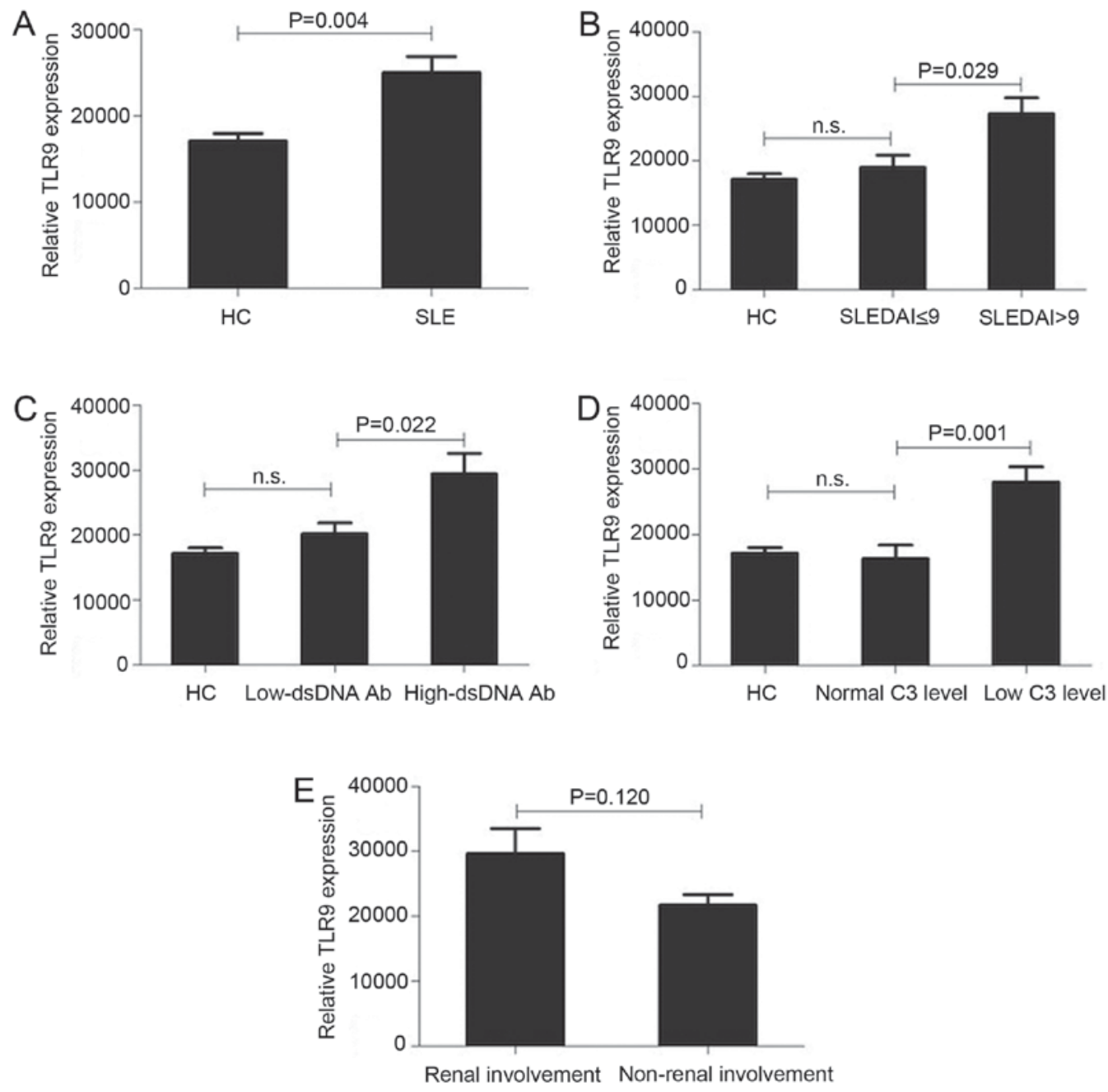

Figure 1. The expression of TLR9 mRNA is increased in whole blood samples from patients with SLE and is associated with SLEDAI scores, anti-dsDNA $\mathrm{Ab}$ levels and C3 levels, but not with renal involvement. (A) Expression of TLR9 mRNA in the whole blood of newly diagnosed patients with SLE (n=90) compared with HCs $(n=49)$. (B) Expression of TLR9 mRNA in the whole blood of patients with SLE in the SLEDAI $\leq 9$ and SLEDAI >9 groups, as well as HCs. (C) Expression of TLR9 mRNA in the whole blood of patients with SLE in the high- and low-dsDNA Ab groups, as well as HCs. (D) Expression of TLR9 mRNA in whole blood from patients with SLE in the normal- and low-C3 level groups, as well as HCs. (E) Expression of TLR9 mRNA in the whole blood of patients with SLE with renal involvement and those with no renal involvement. Data are expressed as the mean \pm standard deviation. TLR9, toll-like receptor 9; HCs, healthy controls; SLE, systemic lupus erythematosus; SLEDAI, SLE Disease Activity Index; C3, complement C3; dsDNA Ab, double stranded DNA antibody titer.

Statistical analysis. Statistical analysis was performed using GraphPad Prism 5.0 (GraphPad Software, Inc., San Diego, CA, USA) and SPSS 20.0 (IBM Corp., Armonk, NY, USA). Categorical variables were analyzed using a Fisher's exact test. Continuous variables were analyzed using a Student's t-test (if the data were normally distributed), a Wilcoxon signed rank test for paired samples or a Mann-Whitney U test for unpaired samples (if the data were not normally distributed). One-way analysis of variance with Tukey's test (for non-parametric data) was used for comparisons among multiple groups. The Kaplan-Meier method was used to analyze the association between TLR9 mRNA expression and poor prognosis of SLE and the log-rank test was used to compare survival curves. A Cox regression model was used to perform multivariable survival analysis. The difference between TLR9 mRNA expression at baseline (time of SLE diagnosis) and after 2 years of immunosuppressive treatment, was compared by paired analysis using the Wilcoxon signed rank test. $\mathrm{P}<0.05$ was considered to indicate a statistically significant difference.

\section{Results}

TLR9 mRNA expression is increased in the whole blood cells of patients with SLE and is significantly different in the SLEDAI, anti-dsDNA Ab and C3 patient subgroups but not in the renal involvement subgroup. A total of 87 female and 3 male patients with SLE with a mean age of $38 \pm 13.9$ years were enrolled in the current study. The healthy control group consisted of 47 females and 2 males. The average age of subjects in the control group at study enrollment was $36.8 \pm 12.8$ years. There were no significant differences in the age or sex of patients with SLE compared with healthy controls.

The expression of TLR9 mRNA in the whole blood samples of the 90 patients with SLE and 49 healthy controls was compared to identify the role of TLR9 in the pathogenesis of SLE. Levels of TLR9 mRNA in patients newly diagnosed with SLE were significantly higher than in healthy controls $(\mathrm{P}=0.004$; Fig. 1A). The baseline demographic and clinical characteristics of patients in the high-TLR9 and low-TLR9 
Table II. Univariate and multivariate analyses of risk factors for SLE prognosis.

\begin{tabular}{lccccccc}
\hline & \multicolumn{3}{c}{ Univariate analysis } & & \multicolumn{2}{c}{ Multivariate analysis } \\
\cline { 2 - 4 } Variable & HR & $95 \%$ CI & P-values & & HR & $95 \%$ CI & P-values \\
\hline Persistent proteinuria of $>0.5$ g per day & 3.600 & $1.879-6.898$ & $<0.0001^{\mathrm{a}}$ & & 6.314 & $2.858-13.947$ & $<0.0001^{\mathrm{a}}$ \\
High-TLR9 level & 3.812 & $1.963-7.400$ & $<0.0001^{\mathrm{a}}$ & & 3.852 & $1.931-7.684$ & $<0.0001^{\mathrm{a}}$ \\
$>1$ immunosuppressant for therapy & 0.775 & $0.401-1.499$ & 0.450 & & 0.374 & $0.173-0.808$ & $0.012^{\mathrm{a}}$ \\
CRP & 1.006 & $1.001-1.012$ & $0.027^{\mathrm{a}}$ & & 1.013 & $1.007-1.019$ & $<0.0001^{\mathrm{a}}$ \\
Serum C3 level & 0.311 & $0.098-0.989$ & $0.048^{\mathrm{a}}$ & & & \\
SLEDAI & 1.051 & $1.016-1.087$ & $0.004^{\mathrm{a}}$ & & & \\
\hline
\end{tabular}

${ }^{a} \mathrm{P}<0.05$. CRP, C-reactive protein; SLEDAI, systemic lupus erythematosus disease activity index; TLR9, toll-like receptor 9; HR, hazard ratio; $\mathrm{CI}$, confidence interval.

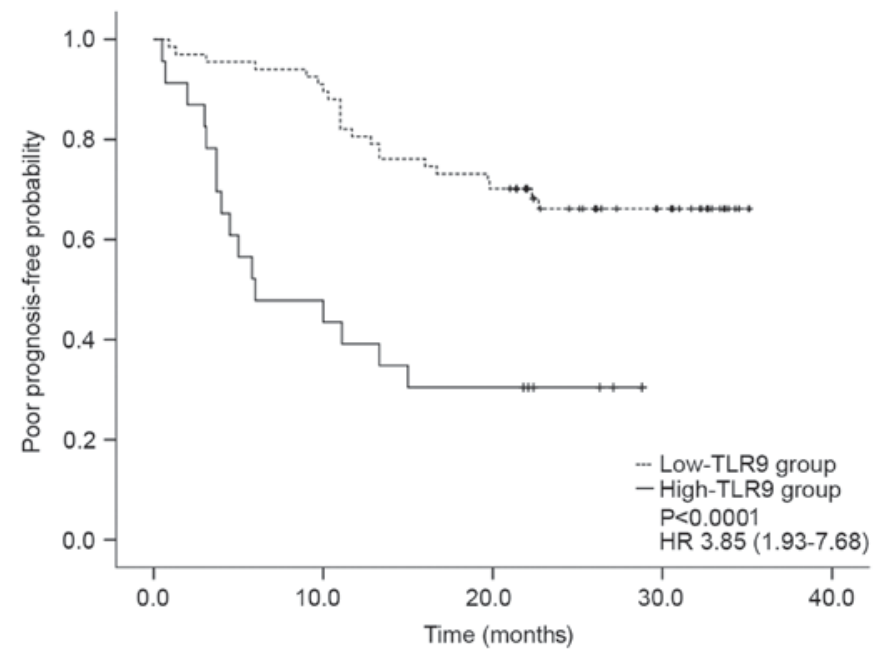

Figure 2. Kaplan-Meier survival curve of SLE prognosis associated with TLR9 mRNA expression. A total of 90 patients with SLE were divided into two groups according to the levels of TLR9 mRNA at SLE onset. Patients with TLR9 $\mathrm{mRNA}$ expression levels higher than the mean TLR9 $\mathrm{mRNA}$ level in the healthy controls +2 standard deviations were placed in the high-TLR9 group and the remaining patients were placed in the low-TLR9 group. SLE, systemic lupus erythematosus; TLR9, Toll-like receptor 9; HR, hazard ratio.

groups as well as the treatments administered are presented in Table I.

Differences in the expression of TLR9 mRNA in the different subgroups of patients with SLE were subsequently determined. Levels of TLR9 mRNA expression in the whole blood samples of patients with SLE were significantly higher in the SLEDAI $>9$ group compared with the SLEDAI $\leq 9$ group $(\mathrm{P}=0.029$, Fig. 1B). Similarly, TLR9 mRNA expression was significantly increased in the high-dsDNA Ab group compared with the low-dsDNA Ab group ( $\mathrm{P}=0.022$; Fig. 1C) and the low-C3 level group compared with the normal-C3 level group ( $\mathrm{P}=0.001$; Fig. 1D). The difference in the levels of TLR9 mRNA in patients with renal involvement compared with patients with no renal involvement was not significant (Fig. 1E). The difference in the percentage of patients with renal involvement (persistent proteinuria of $>0.5 \mathrm{~g} / \mathrm{day}$ ) between the low-TLR 9 and high-TLR9 group was also not significant (Table I).
High TLR9 mRNA expression is independently associated with the poor prognosis of patients with SLE. During the 2-year follow-up period, 52 patients $(57.8 \%)$ experienced clinical remission and the remaining 38 patients $(42.2 \%)$ were categorized into the poor prognosis group. The difference in age, sex, onset-diagnosis lag-time, laboratory analysis findings, other clinical manifestations and the therapies administered to the high and low-TLR9 groups was not significant (Table I). However, there was a significant increase in neuropsychiatric manifestations $(\mathrm{P}=0.029)$, application of cyclosporine $(\mathrm{P}<0.001)$, mortality rates $(\mathrm{P}=0.035)$ and poor prognosis $(\mathrm{P}=0.003)$ in the high-TLR9 group compared with the low-TLR9 group over the 2-year follow-up period (Table I).

Univariate analyses demonstrated that persistent proteinuria $>0.5 \mathrm{~g}$ per day [hazard ratio (HR), 3.81; 95\% confidence interval (CI), 1.96-7.40], serum C3 (HR, 0.31; 95\% CI, 0.10-1.0), SLEDAI (HR, 1.05; 95\% CI, 1.02-1.09), C-reactive protein (HR, 1.006; 95\% CI, 1.001-1.012) and high-TLR9 mRNA levels (HR, 3.60; 95\% CI, 1.88-6.90) were predictive of poor patient prognosis over the 2-year follow-up period (Table II). Following adjustment for several factors, including age, persistent proteinuria ( $>0.5 \mathrm{~g} /$ day), serum C3 serum level, SLEDAI, C-reactive protein, treatment with $>1$ immunosuppressant therapy and high-TLR9 mRNA level, the multivariate analysis demonstrated that persistent proteinuria ( $>0.5 \mathrm{~g} / \mathrm{day}$; HR, 6.314; 95\% CI, 2.858-13.947), C-reactive protein (HR, 1.013; 95\% CI, 1.007-1.019) and high-TLR9 mRNA levels (HR 3.852; 95\% CI, 1.931-7.684) were independent risk factors for poor prognosis, whereas treatment with $>1$ immunosuppressant (HR, 0.374; 95\% CI, 0.173-0.808) was indicative of favorable prognosis (Table II).

The median time of patients with SLE developing poor prognosis was 6 months following diagnosis (range, 0-13.83 months). Kaplan-Meier survival curves indicated that the 2-year poor prognosis-free probability was $66 \%(95 \% \mathrm{CI}$, 54.2-77.8) in the low-TLR9 group and 30\% (95\% CI, 10.4-49.6) in the high-TLR9 group (HR, 3.85; 95\% CI, 1.93-7.68; Fig. 2). This difference between prognosis-free probability was significant $(\mathrm{P}<0.0001$; all Fig. 2).

TLR9 mRNA levels remain high in the poor prognosis group following treatment for 2 years with immune suppressants. At 


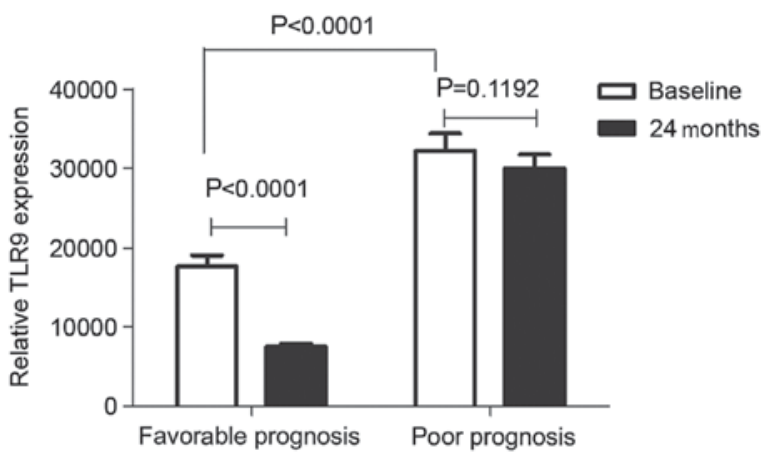

Figure 3. TLR9 mRNA levels remained high in the poor prognosis group in the 2 years following diagnosis of systemic lupus erythematosus. Expression of TLR9 mRNA at the beginning of the study (baseline) and 2 years following diagnosis were compared in the favorable prognosis group $(n=20)$ and poor prognosis group $(n=10)$. There was a significant difference in the expression of TLR9 mRNA between the favorable prognosis and poor prognosis groups at baseline $(\mathrm{P}<0.0001)$. The expression of TLR9 mRNA decreased significantly 2 years following diagnosis $(\mathrm{P}<0.0001)$ in the favorable prognosis group compared with the level at baseline but remained high in the poor prognosis group $(\mathrm{P}=0.1192)$. Data are expressed as the mean \pm standard deviation. TLR9, toll-like receptor 9 .

the end of the 2-year follow-up period, the expression of TLR9 mRNA in the favorable prognosis group $(n=20)$ was compared with the poor prognosis group $(n=10)$ at baseline and 2 years following diagnosis of SLE (Fig. 3). There was a significant increase in the baseline expression of TLR9 mRNA in the poor prognosis group compared with the favorable prognosis group $(\mathrm{P}<0.0001)$. Compared with baseline levels, the levels of TLR 9 mRNA expression 2 years following induction of immunosuppressant treatment decreased significantly in the favorable prognosis group $(\mathrm{P}<0.0001)$ but remained high in the poor prognosis group ( $\mathrm{P}=0.1192$; both Fig. 3$)$.

\section{Discussion}

The current study demonstrated that levels of TLR9 mRNA were significantly increased in the whole blood samples from patients with SLE and that these levels were associated with disease activity. Furthermore, to the best of our knowledge this is the first study to demonstrate that increased expression of TLR9 mRNA indicates the poor prognosis of patients with SLE over a 2-year period. Thus, over the short term at least, TLR9 may predict the successful recovery of patients from SLE without remission and determine the most effective therapeutic strategy for treating patients.

In humans, TLR9 is expressed in the spleen, lymph nodes, tonsils and peripheral blood mononuclear cells $(27,28)$. Previous studies have demonstrated that TLR9 is highly expressed in SLE monocytes, T and B cells $(8,12,29,30)$ and that TLR9 expression in patients with active SLE is increased compared with healthy controls (31). The results of the current study are consistent with this, as they indicate that the expression of TLR9 mRNA in patients with active SLE was significantly increased compared with healthy controls. The current study also measured the expression of TLR9 mRNA in peripheral whole blood cells. TLR9 is expressed by monocytes, T and B cells in patients with SLE; therefore, measuring the expression of TLR9 mRNA in the whole blood may provide a better indication of the in vivo environment and avoids the potential adverse effects of separating cells in collected blood samples.

It has been suggested that a deficiency in the clearance of cellular debris causes the accumulation of extracellular nucleic acids, which may be recognized by TLR9 in patients with SLE (32). This is consistent with the results of the current study, as it was demonstrated that patients with SLE and an increased titer of anti-dsDNA Ab exhibited increased expression of TLR9 mRNA.

The association between TLR9 and different clinical parameters remains controversial. Wong et al (8) demonstrated that there was no correlation between TLR 9 expression and SLEDAI in patients with SLE (mean SLEDAI, 3.13 \pm 2.29 ). By contrast, Nakano et al (33) identified a positive correlation between the expression of TLR9 mRNA and SLEDAI in patients with active SLE. Furthermore, Papadimitraki et al (12) reported a positive correlation between levels of TLR9 expression and anti-dsDNA Ab in the B cells of patients with active SLE. The results of the current study demonstrated that the expression of TLR9 mRNA in whole blood cell samples from patients with newly diagnosed active SLE differed significantly different among the SLEDAI, anti-dsDNA Ab and complement C3 subgroups. This suggests that TLR9 is involved in the pathogenesis of active SLE.

The optimal criteria for predicting SLE remission remain unknown, as the results of previous studies provide differing views $(16,24,34)$. Varying definitions of remission are likely to be the main reason for the divergent results. Unlike other studies that define SLE disease activity as complete remission, serologically active clinically quiescent disease or persistently active disease and flare, the current study categorized SLE outcomes as favorable or poor. At the end of the study, the proportion of patients with favorable prognosis and poor prognosis were 57.8 and $42.2 \%$, respectively. Additionally, the expression of TLR 9 mRNA in the whole blood cells of patients at the onset of SLE was closely associated with prognosis at 2 years. The increased expression of TLR9 mRNA at SLE onset was associated with a poorer outcome of SLE during the 2-year follow-up period. The current study demonstrated that the proportion of patients with poor prognosis was $69.6 \%$ in the high-TLR9 group and $32.8 \%$ in the low-TLR9 group. There were $4(17.4 \%)$ patient mortalities in the high-TLR9 group and $2(3.0 \%)$ in the low-TLR9 group. Thus, the increased expression of TLR9 mRNA may indicate an increased likelihood of mortality in patients with SLE.

Currently, there are no reliable predictors of SLE prognosis and the majority of studies concerning SLE prognosis have been clinical reports. It has been demonstrated that glomerulonephritis, vasculitis and hematological abnormalities are independent risk factors for the absence of clinical remission in patients with SLE (4) and a recent study, demonstrated that renal and neurological involvement decreased the likelihood of remission and favorable prognosis (35). The results of the current study are partially consistent with those from these previous studies, as it was demonstrated that renal involvement is an independent risk factor and increased levels of TLR9 mRNA and C-reactive protein in the whole blood are risk factors for poor prognosis in patients with SLE. The effects of renal involvement (persistent proteinuria of $>0.5 \mathrm{~g} / \mathrm{day}$; HR, 6.31) and high TLR9 mRNA levels (HR, 3.85) on patient 
prognosis were greater than those of C-reactive protein (HR, 1.01). It has been demonstrated that high levels of TLR9 mRNA predict poor outcomes in breast cancer (36); however, to the best of our knowledge, the current study is the first to identify an association between TLR9 expression and SLE prognosis. The expression of TLR9 mRNA was significantly decreased 2 years following the induction of immunosuppressive treatment in the favorable prognosis group but remained unchanged in the poor prognosis group.

Administration of $>1$ type of immunosuppressant for SLE therapy promoted the favorable prognosis of patients with SLE in the current study. Although the administration of multiple immunosuppressants is an acceptable practice, it is necessary to evaluate the side effects of immunosuppressants and their cost-effectiveness in the long term.

The role of TLR9 in SLE pathogenesis remains unclear. A previous study suggested that activation of TLR9 induces the progression of nephritis in MRL-Fas lupus prone mice (37), which may lead to the progression of autoimmune disease. Other studies have demonstrated that TLR9 induces cytokine secretion in B cells and plasmacytoid dendritic cells $(29,38)$. Additionally, DNA-containing immune complexes activate TLR9 in SLE (12) and excessive dsDNA fragments may induce high TLR9 expression in patients with SLE. This is consistent with the results of the current study, which identified that TLR9 expression was positively associated with the SLEDAI score in patients with SLE. Previous studies have indicated that SLE disease activity is the primary factor affecting SLE prognosis in the 5 years following diagnosis $(13,14)$. High expression of TLR9 mRNA is suggestive of a severe autoimmune response in patients with SLE and high SLEDAI (37). A more severe autoimmune response makes recovery more difficult, leading to poorer patient prognosis. This has been confirmed in previous studies, which demonstrated that high SLE disease activity is a factor that predicts poor disease prognosis $(13,14)$. Organ damage may also be a risk factor for poor SLE prognosis, however this was not assessed during the follow-up period in the present study, which is a limitation. Furthermore, the potential role of TLR9 expression in SLE prognosis remains unclear.

In conclusion, TLR9 is involved in the pathogenesis of SLE, associated with disease activity and may predict SLE outcome within a 2-year period. This suggests that the detection of TLR9 mRNA at SLE onset should be used to identify patients with poor prognosis who may require more frequent monitoring in the short-term. Thus, TLR9 may be a useful biomarker for predicting SLE prognosis.

\section{Acknowledgements}

The authors wish to thank the members of the Department of Rheumatology and Immunology in the First Hospital of Jilin University for their contributions to the immunological characterization of the patients. The authors also wish to thank patients and their families for their support and cooperation.

\section{References}

1. O'Neill S and Cervera R: Systemic lupus erythematosus. Best Pract Res Clin Rheumatol 24: 841-855, 2010.
2. Kasitanon N, Magder LS and Petri M: Predictors of survival in systemic lupus erythematosus. Medicine (Baltimore) 85: 147-156, 2006.

3. Ward MM, Pyun E and Studenski S: Causes of death in systemic lupus erythematosus. Long-term followup of an inception cohort. Arthritis Rheum 38: 1492-1499, 1995.

4. Zen M, Iaccarino L, Gatto M, Bettio S, Nalotto L, Ghirardello A, Punzi L and Doria A: Prolonged remission in Caucasian patients with SLE: Prevalence and outcomes. Ann Rheum Dis 74: 2117-2122, 2015.

5. Lloyd W and Schur PH: Immune complexes, complement, and anti-DNA in exacerbations of systemic lupus erythematosus (SLE). Medicine (Baltimore) 60: 208-217, 1981.

6. Kao AH, Navratil JS, Ruffing MJ, Liu CC, Hawkins D, McKinnon KM, Danchenko N, Ahearn JM and Manzi S: Erythrocyte $\mathrm{C} 3 \mathrm{~d}$ and $\mathrm{C} 4 \mathrm{~d}$ for monitoring disease activity in systemic lupus erythematosus. Arthritis Rheum 62: 837-844, 2010.

7. Bootsma H, Spronk PE, Ter Borg EJ, Hummel EJ, de Boer G, Limburg PC and Kallenberg CG: The predictive value of fluctuations in IgM and IgG class anti-dsDNA antibodies for relapses in systemic lupus erythematosus. A prospective long-term observation. Ann Rheum Dis 56: 661-666, 1997.

8. Wong CK, Wong PT, Tam LS, Li EK, Chen DP and Lam CW: Activation profile of Toll-like receptors of peripheral blood lymphocytes in patients with systemic lupus erythematosus. Clin Exp Immunol 159: 11-22, 2010.

9. Wu O, Chen GP, Chen H, Li XP, Xu JH, Zhao SS, Sheng J, Feng JB, Cai J, Fang XH, et al: The expressions of Toll-like receptor 9 and T-bet in circulating B and T cells in newly diagnosed, untreated systemic lupus erythematosus and correlations with disease activity and laboratory data in a Chinese population. Immunobiology 214: 392-402, 2009.

10. Clancy RM, Markham AJ and Buyon JP: Endosomal Toll-like receptors in clinically overt and silent autoimmunity. Immunol Rev 269: 76-84, 2016.

11. Means TK, Latz E, Hayashi F, Murali MR, Golenbock DT and Luster AD: Human lupus autoantibody-DNA complexes activate DCs through cooperation of CD32 and TLR9. J Clin Invest 115: 407-417, 2005.

12. Papadimitraki ED, Choulaki C, Koutala E, Bertsias G, Tsatsanis C, Gergianaki I, Raptopoulou A, Kritikos HD, Mamalaki C, Sidiropoulos P and Boumpas DT: Expansion of toll-like receptor 9-expressing B cells in active systemic lupus erythematosus: Implications for the induction and maintenance of the autoimmune process. Arthritis Rheum 54: 3601-3611, 2006.

13. Cervera R, Khamashta MA, Font J, Sebastiani GD, Gil A, Lavilla P, Mejía JC, Aydintug AO, Chwalinska-Sadowska H, de Ramón E, et al: Morbidity and mortality in systemic lupus erythematosus during a 10-year period: A comparison of early and late manifestations in a cohort of 1,000 patients. Medicine (Baltimore) 82: 299-308, 2003.

14. Abu-Shakra M, Urowitz MB, Gladman DD and Gough J: Mortality studies in systemic lupus erythematosus. Results from a single center. II. Predictor variables for mortality. J Rheumatol 22: 1265-1270, 1995.

15. Abu-Shakra M, Urowitz MB, Gladman DD and Gough J: Mortality studies in systemic lupus erythematosus. Results from a single center. I. Causes of death. J Rheumatol 22: 1259-1264, 1995.

16. Formiga F, Moga I, Pac M, Mitjavila F, Rivera A and Pujol R: High disease activity at baseline does not prevent a remission in patients with systemic lupus erythematosus. Rheumatology (Oxford) 38: 724-727, 1999.

17. Hochberg MC: Updating the American College of Rheumatology revised criteria for the classification of systemic lupus erythematosus. Arthritis Rheum 40: 1725, 1997.

18. Zen M, Bassi N, Nalotto L, Canova M, Bettio S, Gatto M, Ghirardello A, Iaccarino L, Punzi L and Doria A: Disease activity patterns in a monocentric cohort of SLE patients: A seven-year follow-up study. Clin Exp Rheumatol 30: 856-863, 2012.

19. Bombardier C, Gladman DD, Urowitz MB, Caron D and Chang CH: Derivation of the SLEDAI. A disease activity index for lupus patients. The Committee on Prognosis Studies in SLE. Arthritis Rheum 35: 630-640, 1992.

20. Somerfield SD, Roberts MW and Booth RJ: Double-stranded DNA antibodies: A comparison of four methods of detection. J Clin Pathol 34: 1032-1035, 1981. 
21. Haugbro K, Nossent JC, Winkler T, Figenschau Y and Rekvig OP Anti-dsDNA antibodies and disease classification in antinuclear antibody positive patients: The role of analytical diversity. Ann Rheum Dis 63: 386-394, 2004.

22. Ng KP, Manson JJ, Rahman A and Isenberg DA: Association of antinucleosome antibodies with disease flare in serologically active clinically quiescent patients with systemic lupus erythematosus. Arthritis Rheum 55: 900-904, 2006.

23. Gladman DD, Urowitz MB and Keystone EC: Serologically active clinically quiescent systemic lupus erythematosus: A discordance between clinical and serologic features. Am J Med 66: 210-215, 1979.

24. Nikpour M, Urowitz MB, Ibañez D and Gladman DD: Frequency and determinants of flare and persistently active disease in systemic lupus erythematosus. Arthritis Rheum 61: 1152-1158, 2009.

25. Tseng CE, Buyon JP, Kim M, Belmont HM, Mackay M, Diamond B, Marder G, Rosenthal P, Haines K, Ilie V and Abramson SB: The effect of moderate-dose corticosteroids in preventing severe flares in patients with serologically active, but clinically stable, systemic lupus erythematosus: Findings of a prospective, randomized, double-blind, placebo-controlled trial. Arthritis Rheum 54: 3623-3632, 2006.

26. Livak KJ and Schmittgen TD: Analysis of relative gene expression data using real-time quantitative PCR and the 2(-Delta Delta C(T)) method. Methods 25: 402-408, 2001.

27. Nishimura M and Naito S: Tissue-specific mRNA expression profiles of human toll-like receptors and related genes. Biol Pharm Bull 28: 886-892, 2005.

28. Chuang TH and Ulevitch RJ: Cloning and characterization of a sub-family of human toll-like receptors: HTLR7, hTLR8 and hTLR9. Eur Cytokine Netw 11: 372-378, 2000.

29. Rao H, Zeng Q, Liang Y, Xiao C, Xie S and Xu X: Correlation between TLR9 expression and cytokine secretion in the clinical diagnosis of systemic lupus erythematosus. Mediators Inflamm 2015: 710720, 2015.

30. Komatsuda A, Wakui H, Iwamoto K, Ozawa M, Togashi M, Masai R, Maki N, Hatakeyama T and Sawada K: Up-regulated expression of Toll-like receptors mRNAs in peripheral blood mononuclear cells from patients with systemic lupus erythematosus. Clin Exp Immunol 152: 482-487, 2008.
31. Mortezagholi S, Babaloo Z, Rahimzadeh P, Namdari H, Ghaedi M, Gharibdoost F, Mirzaei R, Bidad K and Salehi E: Evaluation of TLR9 expression on PBMCs and CpG ODN-TLR9 ligation on IFN- $\alpha$ production in SLE patients. Immunopharmacol Immunotoxicol 39: 11-18, 2017.

32. Kruse K, Janko C, Urbonaviciute V, Mierke CT, Winkler TH, Voll RE, Schett G, Muñoz LE and Herrmann M: Inefficient clearance of dying cells in patients with SLE: Anti-dsDNA autoantibodies, MFG-E8, HMGB-1 and other players. Apoptosis 15: 1098-1113, 2010.

33. Nakano S, Morimoto S, Suzuki J, Nozawa K, Amano H, Tokano Y and Takasaki Y: Role of pathogenic auto-antibody production by Toll-like receptor 9 of B cells in active systemic lupus erythematosus. Rheumatology (Oxford) 47: 145-149, 2008.

34. Laustrup H, Voss A, Green A and Junker P: SLE disease patterns in a Danish population-based lupus cohort: An 8-year prospective study. Lupus 19: 239-246, 2010.

35. Medina-Quiñones CV, Ramos-Merino L, Ruiz-Sada P and Isenberg D: Analysis of complete remission in systemic lupus erythematosus patients over a 32-year period. Arthritis Care Res (Hoboken) 68: 981-987, 2016.

36. Qiu J, Shao S, Yang G, Shen Z and Zhang Y: Association of Toll like receptor 9 expression with lymph node metastasis in human breast cancer. Neoplasma 58: 251-255, 2011.

37. Anders HJ, Vielhauer V, Eis V, Linde Y, Kretzler M, Perez de Lema G, Strutz F, Bauer S, Rutz M, Wagner H, et al: Activation of toll-like receptor-9 induces progression of renal disease in MRL-Fas (lpr) mice. FASEB J 18: 534-536, 2004.

38. He B, Qiao X and Cerutti A: CpG DNA induces IgG class switch DNA recombination by activating human B cells through an innate pathway that requires TLR9 and cooperates with IL-10. J Immunol 173: 4479-4491, 2004. 